\title{
Resolution of the Osmosedimentation Equations by Numerical Methods
}

\begin{abstract}
A.O. FORTUNA, Departamento de Ciências de Computação e Estatística, Instituto Ciências Matemáticas e de Computação, Universidade de São Paulo, Cx.P. 668, 13560-970 São Carlos, SP, Brazil
\end{abstract}

C.R. NETO, Laboratório Associado de Computação e Matemática Aplicada, Instituto Nacional de Pesquisas Espaciais, Cx.P. 515, 12201-970 São José dos Campos, SP, Brazil

M.A. TEnAN, Centro de Ciências Exatas e de Tecnologia, Universidade de Mogi das Cruzes, Av. Dr. Cândido Xavier de Almeida Souza 200, 08780-911 Mogi das Cruzes, SP, Brazil.

\begin{abstract}
The osmosedimentation process is an example of spatiotemporal patterns formation in system driven away from equilibrium, a subject of great interest nowadays. Here, the osmosedimentation process is numerically modeled by the incompressible Navier-Stokes equations with appropriate source terms, together with a scalar transport equation for the solute concentration. The unsteady NavierStokes equations are written in primitive variable form, and solved using a coupled point solver. Second order accuracy in time is enforced through the use of a second order backward differencing discretization of the time derivative. The convective terms are modeled using a high order bounded scheme that reduces numerical diffusion and does not create spatially-oscillatory solutions. We were able to gain a better understanding of the far from equilibrium pattern formation in osmosedimentation processes and to extend previous numerical simulation farther in time.
\end{abstract}

\section{Introduction}

One of the most interesting examples of non-equilibrium pattern formation is that given by the well-known Rayleigh-Bénard convection problem. This problem is concerned with the flow of a thin horizontal fluid layer heated from below [1]: for low values of the temperature gradient, fluid motion is inhibited by viscosity, however, if the temperature gradient exceedes a critical value, buoyancy overcomes viscous resistance and the fluid layer resolves itself into a number of cells. This means that a spatial structure comes into being spontaneously, or in other words, the system undergoes self-organization. 
The main characteristic of this effect is that the external influence (combination of the adverse temperature gradient and gravity) is a global influence that just stimulates the start of internal mechanisms capable of producing internal organization.

The external influence does not contain by itself any directions as to whether the system should display any macroscopic structure. In the present work the problem is extended from pure fluids to fluid mixtures. The convection cell patterns are now induced by composition inhomogeneities of the fluid system rather than by temperature inhomogeneities.

We investigate the problem of fluid flow and mass transport in a viscous binary fluid mixture under osmosedimentation, $i$.e. the sedimentation process that takes place under a strong centrifugal field in a dialysis cell $[2,3,4,5,6]$. For the present study we improve our model proposed earlier [7] by introducing a Boussinesq correction term $[1,8]$ to the Navier-Stokes equations which govern the fluid motion. These equations are then solved numerically.

\section{The Model}

The system consists of a dialysis cell under a strong centrifugal field [7]. A semipermeable membrane divides longitudinally the cell into two compartments, one of which contains a viscous binary ideal solution of initially uniform composition, and the other is filled with pure solvent. The semipermeable membrane is located at $x=0 \mathrm{~cm}$ (see figure 2 , where only the viscous binary ideal solution in the right side of the osmosedimentation cell is showed).

Thanks to osmotic forces as well as strong inertial forces fluid flow is stablished within the cell. For the details of the model - balance equations, boundary conditions and numerical values for the parameters - we refer to [7]. Differently from our previous simplified model, the small density non-uniformities generated in the solution by fluid circulation plays an important role in the overall mass transfer process. In fact, the Boussinesq term in the momentum equation is capable of imposing significant mass motions in the bulk of the solution. Convection cells are superimposed to the fluid flow from top to bottom of the solution compartment, and become dominant. These fluid motions are thus responsible for solute mass redistribution in the solution half-cell.

\section{Governing Equations}

The physical model required the solution of the following coupled set of equations [9]. The Navier-Stokes equations for incompressible flow, which in vector form can be written as:

$$
\begin{aligned}
\frac{\partial \vec{V}}{\partial t}+\nabla \cdot(\vec{V} \vec{V}) & =-\frac{\nabla p}{\rho}+\nu \nabla^{2} \vec{V}+\left[1-\xi\left(\chi_{1}-\bar{\chi}\right)\right] \vec{G} \\
\nabla \cdot \vec{V} & =0
\end{aligned}
$$

where $p$ is the pressure, $\vec{V}=(u \hat{x}, v \hat{z})$ is the velocity vetor, $\vec{G}=w^{2} r \hat{z}$ is the inertial field, and $\rho$ and $\nu$ are the density and kinematic viscosity of the fluid, respectively. 
The linear approximation constant $\xi$ is given by $\xi=-\left(\frac{1}{\rho} \frac{\partial \rho}{\partial \chi_{1}}\right)_{\bar{\chi}}$, where $\chi_{1}$ stands for the solute molar fraction and $\bar{\chi}$ for the initial homogeneous solute molar fraction.

The convection-diffusion equation for the solute molar fraction $\chi_{1}$ of the solute is

$$
\frac{\partial \chi_{1}}{\partial t}=-\nabla \cdot\left(\chi_{1} \vec{V}+\vec{j}_{1}\right)
$$

where $\vec{j}_{1}$ is the flux density vector of the solute. For an ideal mixture the flux density of the solute is [9]:

$$
\vec{j}_{1}=\frac{c^{2}}{\rho} M_{0} M_{1} D\left[\nabla \chi_{0}+\frac{M_{0} \chi_{0}}{R T}\left(\frac{V_{0}}{M_{0}}-\frac{1}{\rho}\right) \nabla p\right],
$$

where $c$ is molar density of solution, $M_{1}$ is the molecular weight of solute, $D$ is the solute diffusion coefficient, $\chi_{0}$ is the solvent molar fraction, $R$ is the gas constant, $T$ is the absolute temperature and $V_{0}$ is the solvent partial molal volume.

\section{Initial and Boundary Conditions}

The fluid is assumed to be initialy at rest everywhere in the domain, and all the boundary conditions for the discretised Navier-Stokes equations are imposed on the velocity components only. Both $u$ and $v$ at solid walls are set to zero. At the membrane there is only the normal velocity component $u$.

As the initial condition for the density it is imposed that $\rho_{1}$ are uniform everywhere in the domain. Diffusion of solute across solid boundaries is prevented by setting the appropriate gradients to zero. At the membrane, the following boundary condition is adopted, $j_{0}=-K_{m} \Delta \mu_{0, \alpha \beta}$, where $K_{m}$ is the membrane permeability and $\Delta \mu_{0, \alpha \beta}$ is given by the difference between the solvent chemical potentials across the membrane [5].

\begin{tabular}{|c|c|}
\hline $\mathrm{T}(\mathrm{K})=277$ & $\mathrm{M}_{0}(\mathrm{~g} / \mathrm{mol})=18$ \\
\hline$\mu_{0}(\mathrm{P})=1.57 \times 10^{-2}$ & $\mathrm{M}_{1}(\mathrm{~g} / \mathrm{mol})=3.6 \times 10^{4}$ \\
\hline $\mathrm{D}_{01}\left(\mathrm{~cm}^{2} / \mathrm{s}\right)=10^{-6}$ & $\rho_{0}\left(\mathrm{~g} / \mathrm{cm}^{3}\right)=1.0$ \\
\hline $\mathrm{L}_{0}(\mathrm{~cm})=1.0$ & $\mathrm{~V}_{0} / \mathrm{M}_{0}\left(\mathrm{~cm}^{3} / \mathrm{g}\right)=1.0$ \\
\hline $\mathrm{L}_{z}(\mathrm{~cm})=7.5$ & $\rho\left(\mathrm{g} / \mathrm{cm}^{3}\right)=1.0033$ \\
\hline $\mathrm{R}_{0}(\mathrm{~cm})=15.7$ & $\mathrm{c}\left(\mathrm{mol} / \mathrm{cm}^{3}\right)=5.52 \times 10^{-2}$ \\
\hline$\omega(\mathrm{rpm})=2000$ & $\mathrm{x}_{1}($ initial $)=5.0 \times 10^{-6}$ \\
\hline
\end{tabular}

\section{The Numerical Method}

Equation (2.1) is discretized on a staggered grid [10] using finite differences. Equation (2.2) is discretized using the finite volume technique. Viscous terms are discretised using standard second order centred differences, e.g.,

$$
\left(\frac{\partial^{2} \phi}{\partial x^{2}}\right)=\frac{u_{i-1, j}-2 u_{i, j}+u_{i+1, j}}{(\Delta x)^{2}}+O(\Delta x)^{2} .
$$


When close to domain boundaries, derivatives are discretised using appropriate non-centred formulæ:

$$
\frac{\partial^{2} u}{\partial x^{2}}=\frac{u_{i, j+1}-3 u_{i, j}}{\frac{3}{4}(\Delta x)^{2}}+O(\Delta x) .
$$

In order to avoid the time step limitations characteristic of explicit integration schemes, an implicit discretisation of the spatial terms is adopted. Since timeaccurate solutions were important for this problem, a time-stepping is performed with a second order backward differencing given by

$$
\frac{\partial \phi}{\partial t}=\frac{3 \phi^{n+1}-4 \phi^{n}+\phi^{n-1}}{2(\Delta t)}+O(\Delta t)^{2},
$$

where $\phi$ is either component of the velocity (in the Navier-Stokes equations) or $\chi_{1}$ in the convection-diffusion equation.

To correctly model the convection effects present in the flow, we employed Zhu's HLPA non-diffusive, bounded scheme [11, 12]. The implementation followed the defered correction technique: HLPA's discretization is treated explicitly and as a source term in the momentum and convection-diffusion equations. Their convective terms were discretized using first order upwind. An equivalent term was subtracted as an explicit source term, so that when the iterative methods converged for a given time step, both upwind discretizations cancelled out and only HLPA remained. The deferred correction retained the diagonal dominance of the coefficient matrix while at the same time, providing a low-diffusion alternative to the first order upwind scheme.

The net result of the discretisation procedure is a coupled system of linear equations which must be solved at each time step. Since there is no evolution equation for the pressure, an auxiliary scheme must be employed for the computation of the pressure field within the domain.

\section{Solution Procedure}

The solution of the discretised fluid flow equations is obtained via a relaxation method similar to the Gauss-Seidel procedure; the method employed is known as Symmetric-Coupled Gauss-Seidel (SCGS) [13].

Each four velocities and pressure on the staggered grid constitute a cell (see figure 1). Following the SCGS method, let us define a correction vector $\delta$ whose elements will correct the four velocities and the pressure, therefore satisfying the discrete versions of equations (2.1) and (2.2) simultaneously. Thus,

$$
\begin{aligned}
u_{i-1 / 2, j}^{n+1,(k+1)} & =u_{i-1 / 2, j}^{n+1,(k+1 / 2)}+\delta u_{i-1 / 2, j}, \\
u_{i+1 / 2, j}^{n+1,(k+1 / 2)} & =u_{i+1 / 2, j}^{n+1,(k)}+\delta u_{i+1 / 2, j}, \\
v_{i, j-1 / 2}^{n+1,(k+1)} & =v_{i, j-1 / 2}^{n+1,(k+1 / 2)}+\delta v_{i, j-1 / 2},
\end{aligned}
$$




$$
\begin{aligned}
v_{i, j+1 / 2}^{n+1,(k+1 / 2)} & =v_{i, j+1 / 2}^{n+1,(k)}+\delta v_{i, j+1 / 2}, \\
p_{i, j}^{(k+1)} & =p_{i, j}^{(k)}+\delta p_{i, j},
\end{aligned}
$$

where $n$ and $k$ are the time and iteration level indices, respectively. The value $k+1 / 2$ appears because all but the boundary velocities are corrected twice during a single sweep of the SCGS method. The SCGS is a coupled solution scheme.

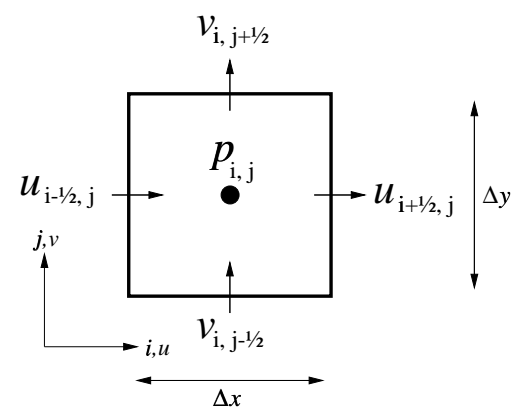

Figure 1: Staggered grid cell.

Substituting equations (4.1) into the discretized momentum equations results in the following linear system:

$$
\left[\begin{array}{ccccc}
\frac{I_{i-1 / 2, j}^{u}}{\omega_{1}} & 0 & 0 & 0 & \frac{\Delta t}{\rho(\Delta x)} \\
0 & \frac{I_{i+1 / 2, j}^{u}}{\omega_{1}} & 0 & 0 & \frac{-\Delta t}{\rho(\Delta x)} \\
0 & 0 & \frac{I_{i, j-1 / 2}^{v}}{\omega_{2}} & 0 & \frac{\Delta t}{\rho(\Delta y)} \\
0 & 0 & 0 & \frac{I_{i, j+1 / 2}^{v}}{\omega_{2}} & \frac{-\Delta t}{\rho(\Delta y)} \\
\frac{-1}{\Delta x} & \frac{1}{\Delta x} & \frac{-1}{\Delta y} & \frac{1}{\Delta x} & 0
\end{array}\right]\left[\begin{array}{c}
\delta u_{i-1 / 2, j} \\
\delta u_{i+1 / 2, j} \\
\delta v_{i, j-1 / 2} \\
\delta v_{i, j+1 / 2} \\
\delta p_{i, j}
\end{array}\right]=\left[\begin{array}{c}
R_{i-1 / 2, j}^{u} \\
R_{i+1 / 2, j}^{u} \\
R_{i, j-1 / 2}^{v} \\
R_{i, j+1 / 2}^{v} \\
R_{i, j}^{c}
\end{array}\right],
$$

which when solved, provides the required values for the corrections. The elements of the RHS vector of the above system are the residuals from the momentum and continuity equations. Relaxation parameters $\omega_{1}$ and $\omega_{2}$ are taken to be equal to $\omega \approx 0,5$.

SCGS requires the solution of system (4.2) for every cell in the domain. Because of the banded structure of the coefficient matrix, it can be partioned and solved efficiently using two explicit formulæ.

The convection-diffusion equation is solved by applying the line version of the standard Gauss-Seidel iterative method. The resulting tridiagonal system is solved for every line using the Thomas algorithm.

For each time step, the iterative procedure is repeated until the residuals of discrete versions of equations (2.1) and (2.2) fall below a certain threshold value $\epsilon$. This is a measure of how closely the numerical values satisfy the discretized equa- 
tions. The value of $\epsilon$ was determined through numerical experiments and eventually set at $10^{-4}$.

\section{Results and Discussion}

Fig. 2 shows the simulation results of the model for the stream lines at different times. In Fig. 3 we see the center of the eddies in the osmosedimentation cell. In the bulk of the osmosedimentation cell, to maintain the momentum conservation, eddies are generated in pairs of clockwise and counter-clockwise rotation. Sometimes, eddies from opposite rotations collide and disappear. There are also lone eddies at the top and bottom walls.

(a)

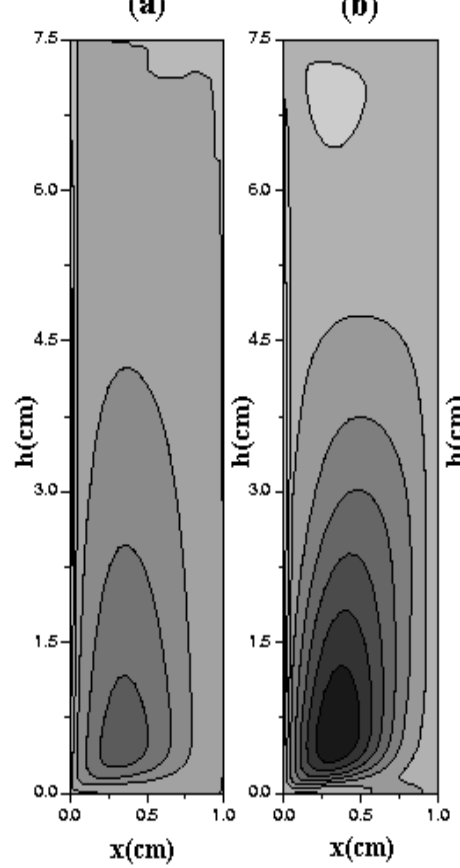

(c)

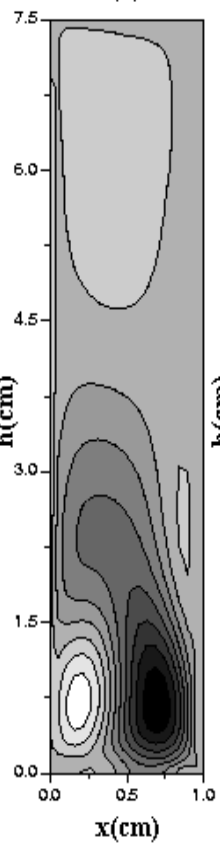

(d)

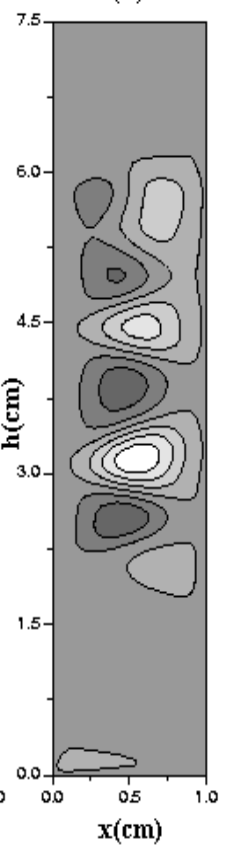

Figure 2: Streamlines of the osmosedimentation process at different simulation steps. From left to right (a) $n=63$, (b) $n=75$, (c) $n=125$ and (d) $n=650$. White (black) cells are clockwise (counter-clockwise) convection cells. The streamlines values are in arbitrary scale.

In general, as shown in Fig. 3, the eddies move to the bottom of the cell. Due to this convective flow, the osmosedimentation process provides a mass extratification [14] that is unstable under centrifugation. When there is enough unstable mass depletion under a given mass concentration, the counter-clockwise eddies raise, 


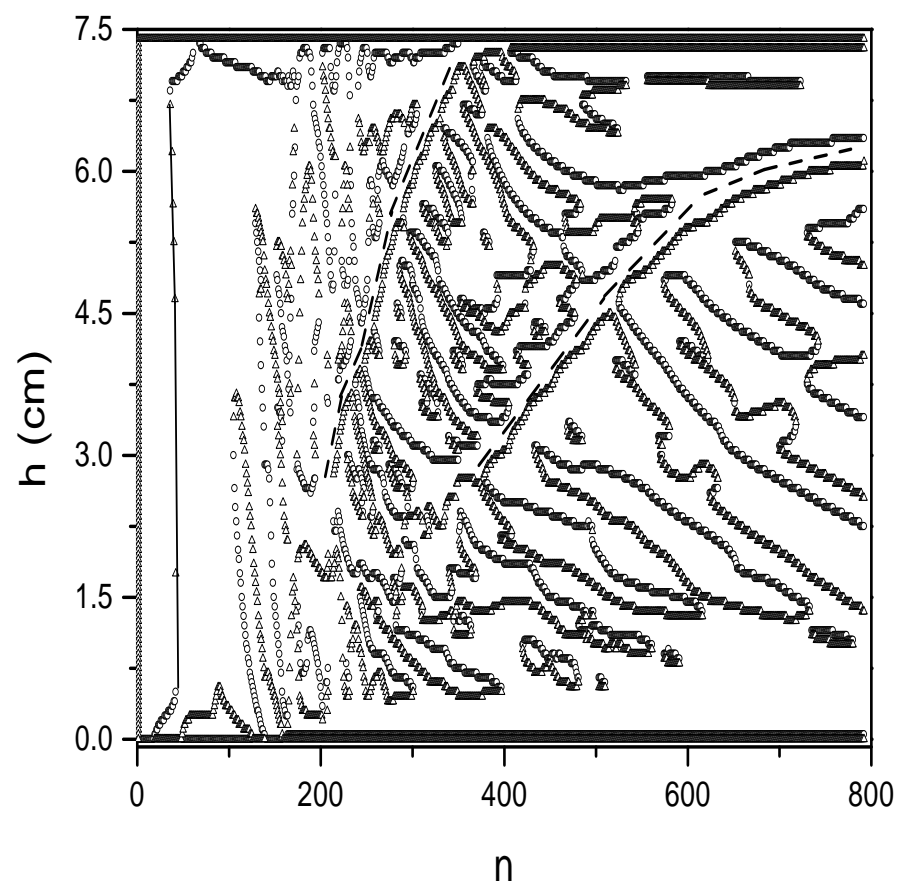

Figure 3: Bifurcation diagram of the onset of convection cells in the osmosedimentation process. The vertical axis gives the position of the eddies center against time observed at $x=0.25 \mathrm{~cm}$ from the membrane. The $\bigcirc(\triangle)$ are the center of the clockwise (counter-clockwise) eddies. The two generation lines are indicated by the dash lines.

clearly defining two generation lines (dash lines in Fig. 3). At this point there is a mass redistribution which tends to diminish non-uniformities in mass distribution. These results are in qualitative agreement with those determined by the previous researchers $[2,3,4,5,6]$.

\section{Conclusions}

The osmosedimentation process has a very complex dynamics and, as shown by $[7,14]$, is a difficult process to model and simulate. Thus, to reduce the risk of instabilities, coupled solvers were employed. The use of a bounded and low-difusivity convection scheme prevents the appearence of false diffusion that could invalidate the results.

Good agreement with previous experiments encourage us to push forward this investigation and study the sensitivity of the dynamics evolution of the osmosedimentation process to changes in the physical parameters such as cell dimensions, 
membrane effects and initial conditions.

\section{Acknowledgements}

The research of the author C.R. Neto was supported by FAPESP and the author M.A. Tenan is grateful to FAEP/OMEC for financial support.

\section{References}

[1] S. Chandrasekhar, "Hydrodynamic and Hydromagnetic Stability", Oxford University Press, London, 1961, Chap. II.

[2] F. Galembeck, P.R. Robillota, E.A. Pinheiro, I. Jokes and N. Bernardes, J. Phys. Chem., 84 (1980), 112.

[3] A.T.N. Pires, S.P. Nunes and F. Galembeck, J. Colloid Interface Sci., 98 (1984), 489.

[4] C.A. Mello, Master Thesis, IQ - UNICAMP, Brazil, 1984.

[5] F. Galembeck and A.T.N. Pires, Separation and Purification Methods, 15 (1986), 97.

[6] S.P. Nunes and F. Galembeck, J. Non-Equilib. Thermodyn., 12 (1987), 205.

[7] C. Rodrigues Neto, M.A. Tenan, J. Non-Equilib. Thermodyn., 19 (1994), 157.

[8] H.J. Kreuzer, "Nonequilibrium Thermodynamics and its Statistical Foundations", Clarendon Press, Oxford, 1981.

[9] R.B. Bird, Theory of diffusion, in "Advances in chemical engineering", (J.W. Hoopes, ed.), vol. I, Academic Press, New York, 1956, 165-174.

[10] R. Peyret and T. D. Taylor, "Computational Methods for Fluid Flow", Springer-Verlag, 1990.

[11] V. Armenio, An improved MAC method (SIMAC) for unsteady high-Reynolds free surface flows, International Journal of Numerical Methods in Fluids, 24 (1997), 185-214.

[12] J. Zhu, On the higher-order bounded discretization schemes for finite volume computations of incompressible flows, Computational Methods in Applied Mechanical Enginnering, 98 (1992), 345-360.

[13] S.P. Vanka, Block-implicit Multigrid Solution of Navier-Stokes Equations in Primitive Variables, Journal of Computational Physics, 65 (1986), 138-158.

[14] C. Rodrigues Neto and M.A. Tenan, Fluid circulation and solute distribution in a binary fluid mixture: Self-organization under osmosedimentation, PHYSICA A, 257 (1998), 536-541. 